\title{
LA EVALUACIÓN DE LA ACCIÓN DOCENTE DESDE UN ENFOQUE METACOGNITIVO
}

\section{The teacher's assesment in coexistence from a metacognitive approach}

\section{A avaliação da ação docente, a partir de um enfoque metacognitivo}

\begin{abstract}
Alejandra Alexia Díaz Pino
(1) Ces Don Bosco-Universidad Complutense de Madrid, España. Teléfono: +34 9145004 72. Correo electrónico: alejandradp@,cesdonbosco.com
\end{abstract}

\section{Resumen}

La metacognición, entendida como la identificación y regulación del conocimiento propio, posibilita un proceso de autoevaluación formativa útil para que el docente pueda valorar su actuación en ámbitos complejos como la convivencia escolar. Este enfoque resulta poco habitual en la formación del profesor o profesora y requiere de guías de actuación que es necesario definir y consensuar, siendo prioritario realizar estudios, como el presente, cuyo objetivo sea identificar y analizar el impacto de los métodos empleados en investigaciones destinadas a la formación metacognitiva docente. Los resultados muestran que el aprendizaje basado en problemas, a través de técnicas de simulación y estrategias de autoevaluación, conforman la metodología comúnmente empleada, pero el número de investigaciones enfocadas a este fin, el área en que se implementan y las características del diseño de investigación presentan una limitación para establecer conclusiones sólidas sobre su pertinencia en la formación en convivencia.

Palabras clave: Metacognición; docente; autoevaluación formativa; convivencia escolar

\begin{abstract}
Metacognition, understood as the identification and regulation of own knowledge, makes possible a very useful formative self-assessment process so that the teacher can value his performance in complex areas such as school coexistence. This approach is unusual in the training of the teacher and requires action guidelines that need to be defined and agreed upon, with a priority to carry out studies, such as the present one, whose objective is to identify and analyze the impact of the methods used in previous
\end{abstract}


research for the initial and continuous teacher training. The results show that problembased learning, through simulation techniques and self-assessment strategies, form the methodology commonly used, but the number of investigations focused on this purpose, the area in which they are implemented and the characteristics of the research design present a limitation to establish solid conclusions on its pertinence in the formation in coexistence.

Keywords: Metacognition; teacher; formative self-assessment; school coexistence

\section{Resumo}

A metacognição, entendida como a identificação e regulação do conhecimento próprio, possibilita um processo de autoavaliação útil para que os docentes possam valorar a sua ação em contextos complexos como a convivência escolar. Este foco é pouco habitual na formação do profesor ou professora e requere guias de atuação que são necessários definir e acordar. Desse modo, é prioritário realizar estudos como este, em que o objetivo passa por identificar e analisar o impacto dos métodos utilizados nas investigações destinadas à formação metacognitiva docente. Os resultados mostram que a aprendizagem baseada em problemas, através de técnicas de simulação e estratégias de autoavaliação compõem a metodologia normalmente utilizada. No entanto, o número de investigações realizadas com este objetivo nesta área e as características do desenho de investigação apresentam limitações para estabelecer conclusões sólidas sobre a sua pertinência na formação em convivência.

Palavras-chave: Metacognição; professora; autoavaliação formativa; convivência escolar

\section{Introducción}

El fomento de una convivencia positiva en la escuela es un proceso de búsqueda de relaciones satisfactorias que implica la reflexión crítica sobre los factores que tienen una influencia notable en la misma (Uruñuela, 2016). En este sentido, el análisis del comportamiento propio por parte del docente resulta relevante, puesto que puede tener una gran repercusión en la convivencia escolar $\mathrm{y}$, especialmente, en las concepciones del estudiante sobre lo que esta significa. El desarrollo de iniciativas basadas en la formación metacognitiva docente parecen especialmente útiles para promover dicho análisis, ya que posibilitan la identificación de aquello que se conoce y cómo se utiliza a 
través de un proceso de autoevaluación formativa continua, tal y como recomienda gran parte de la comunidad científica (Andrade \& Valtcheva, 2009). Consecuentemente, parece pertinente realizar estudios, como el que se presenta a través de estás líneas, cuyo objetivo sea identificar y analizar el impacto de metodologías potencialmente adecuadas para este tipo de formación.

\section{Método}

Se realiza una revisión bibliográfica de todos los estudios disponibles en español e inglés que se destinan a diseñar y poner a prueba programas dirigidos a la formación metacognitiva inicial de estudiantes de Grado en Magisterio y Máster de Educación Secundaria, así como a la formación continua de docentes en activo. Dicha revisión se lleva a cabo a través de la base de datos de la Universidad Complutense de Madrid "BUCea" y se basa en los siguientes criterios de búsqueda: metacognición, docente, profesor, metodología, metacognición, teacher y methodology.

\section{Resultados y discusión}

El número de estudios que se destinan al fomento de competencias metacognitivas por parte del profesor o profesora son escasos y se restringen, fundamentalmente, a la formación metacognitiva del docente en el área de ciencias (Abd-El-Khalick \& Akerson, 2009). El método más utilizado en dichos estudios es el aprendizaje basado en problemas (ABP) cuyo objetivo es presentar situaciones complejas que se pueden dar en el aula y proponer al sujeto la búsqueda de soluciones.

Las situaciones problemáticas, generalmente, se representan en vídeos (Kale \& Whitehouse, 2012) y facilitan que el docente pueda enfrentarse a la resolución de problemas mediante técnicas de dramatización (Franky, 2009) y, lo que es más importante, potencian que el maestro o maestra analice el curso y resultado de su acción a través de estrategias de evaluación basadas en autointerrogación y discusión metacognitiva. Esta es una forma de reflexionar sobre el conocimiento práctico y analizar la eficacia de los procedimientos empleados, así como identificar futuras líneas de actuación (véase: Kramarski \& Kohen ,2017; Leijen et al., 2015; Mevarech \& Kramarski, 1997) con la diferencia, respecto a otros ámbitos, de que en educación no suele haber una única forma de enfrentar un problema, por lo que no hay respuestas únicas (Lin, Schwartz, \& Hatano, 2005). 
Finalmente, desde un punto de vista cuantitativo, es importante destacar que debido a las características del diseño de investigación empleado en estos estudios, especialmente en lo que se refiere al instrumento de evaluación y la muestra, resulta complejo establecer conclusiones claras sobre el impacto de las intervenciones que se han descrito en el desarrollo metacognitivo del docente. No obstante, desde una perspectiva cualitativa, parece relevante subrayar que, aunque el maestro o maestra inicialmente no está familiarizado con este tipo de formación, tras participar en la misma destaca su utilidad en la acción docente eficaz y se muestra dispuesto a seguir utilizando lo aprendido en el aula (véase: Ben-David \& Orion, 2013).

\section{Conclusiones}

Aunque generalmente la formación metacognitiva del docente se lleva a cabo con profesores y profesoras de ciencias y el número de estudios destinados a analizar su utilidad son escasos, siendo esta una limitación sustancial del presente estudio, la metodología en que se basa la misma podría resultar especialmente útil para la formación inicial de docente, especialmente de estudiantes de grado en Magisterio y máster de Educación Secundaria, puesto que ofrece una gran oportunidad para que estos puedan tener un papel activo en la resolución de problemas en áreas complejas como la convivencia y analizar sus actuaciones a través de un proceso de autoevaluación formativa. Se trata, por tanto, de un enfoque que es necesario tener en cuenta y poner a prueba esta área a través de estudios empíricos, muestras representativas e instrumentos fiables que contribuyan a arrojar luz sobre la eficacia de estas propuestas.

\section{Referencias}

Abd-El-Khalick, F., \& Akerson, V. (2009). The influence of metacognitive training on preservice elementary teachers' conceptions of nature of science. International Journal of Science Education, 31(16), 2161-2184. doi:10.1080/09500690802563324

Andrade, H., \& Valtcheva, A. (2009). Promoting learning and achievement through self-assessment. Theory into practice, 48 (1), 12-19.

Ben-David, A., \& Orion, N. (2013). Teachers' voices on integrating metacognition into science education. International Journal of Science Education, 35 (18), 31613193. 
Franky, G. A. (2009). Potencialidades pedagógicas de los entornos de simulación, desde la perspectiva de la cognición situada. TED: Tecné, Episteme y Didaxis, 25, 6271.

Kale, U., \& Whitehouse, P. (2012). Structuring video cases to support future teachers' problem solving. Journal of Research on Technology in Education, 44 (3), 177204. doi:10.1080/15391523.2012.10782586

Kramarski, B., \& Kohen, Z. (2017). Promoting preservice teachers' dual self-Regulation roles as learners and as teachers: Effects of generic vs. specific prompts. Metacognition and Learning, 12 (2), 157-191. doi: 10.1007/s11409-016-9164-8

Leijen, Ä., Allas, R., Pedaste, M., Knezic, D., Mena, J. J., Meijer, P., Husu, J., Krull, E \& Toom, A. (2015). How to support the development of teachers' practical knowledge: Comparing different conditions. Procedia-Social and Behavioral Sciences, 191, 1205-1212. doi: 10.1016/j.sbspro.2015.04.455.

Lin, X., Schwartz, D. L., \& Hatano, G. (2005). Toward teachers' adaptive metacognition. Educational Psychologist, 40 (4), 245-255.

Mevarech, Z. R., \& Kramarski, B. (1997). IMPROVE: A multidimensional method for teaching mathematics in heterogeneous classrooms. American Educational Research Journal, 34 (2), 365-394

Uruñuela, P. M. (2016). Trabajar la convivencia en los centros educativos. Una mirada al bosque de la convivencia. Madrid: Narcea. 